\title{
"Esse est Deus". Los tres "momentos" lógicos del esse creado en el Prólogo a la Obra de las proposiciones de Meister Eckhart
}

\author{
SOFÍA CASTELLO* \\ Universidad de Buenos Aires (Argentina) \\ soficlc@gmail.com
}

\begin{abstract}
Resumen
El presente trabajo tiene como objeto exponer el despliegue que Meister Eckhart realiza del esse creado en el Prólogo a la Obra de las proposiciones. En primer lugar, presentamos el orden de la realidad propuesto por el Dominico, que presenta al esse como la esfera de la representación conceptual del mundo creado. De esta manera, podemos explicar cómo el esse es Dios ("esse est Deus") aunque, al identificarlo como efecto, sea distinto a Dios (causa). En segundo lugar, luego de exponer la estructura general de la realidad, atendemos al movimiento dentro de la esfera del mundo. Y allí encontramos tres "momentos": el esse absolutum mismo, el ente, como representación de la individualidad conceptual y el ente hoc, como la entidad que se despliega en el plano finito. De esta manera, buscamos continuar ampliando los estudios de las particularidades del pensamiento eckhartiano.
\end{abstract}

Palabras clave: Meister Eckhart; mundo creado; ser; orden de la realidad; causa y efecto.

\section{"Esse est Deus". The three logical "moments" of created esse in the Prologue to the Work of Propositions of Meister Eckhart}

\begin{abstract}
In this article we intend to present the three logical "moments" of the created esse that are identifiable in his Prologue to the Work of Propositions'. Firstly, we present the main structure of reality, which positions the esse as the sphere of conceptual representation of the created world. In this way, we can explain how the esse is God ("esse est Deus") even though, as effect, it's not God -understanding "God" as cause. Secondly, after presenting the general structure of reality, we present the movement within the sphere of the esse. And there we can find three "moments": the esse itself, the esse absolutum, as the grounding of the world; the ente, as the representation of conceptual individuality; and the ente hoc, as the creature. This way, we seek to continue expanding the studies of the particularities of Eckhart's thought.
\end{abstract}

Key words: Meister Eckhart; created world; being; order of reality; cause and effect.

\footnotetext{
* Becaria doctoral en Filosofía Medieval por la Universidad de Buenos Aires. Docente de Introducción al Pensamiento Científico en la misma Universidad.
} 


\section{INTRODUCCIÓN}

Hasta hace no demasiado tiempo Meister Eckhart, autor alemán perteneciente a la orden Dominica de fines del s. XIII y principios del s. XIV, era asociado cuasi únicamente con su obra alemana. Sus tratados y sermones en lengua vernácula eran exponentes de la mística renana de su tiempo. Luego, cuando en la primera mitad del s. XX paulatinamente comenzó a descubrirse y editarse su obra latina, se la consideró meramente una suerte de "obligación profesional", una demanda que tuvo que cumplir por su rol en la orden dominica. Por ello, esta parecía asistemática, al no responder a los "verdaderos" intereses del dominico, y, así, se le restaba valor a la obra latina por no ser lo que genuinamente Eckhart "había querido escribir"1.

El hecho de que en el Prólogo general a la Obra tripartita haya presentado un gran marco sistemático de obras —en donde habría de analizar proposiciones generales, sosteniendo que serían mil proposiciones y más, varias cuestiones y, finalmente, exposiciones referidas a pasajes tanto del Antiguo como del Nuevo testamento- ${ }^{2}$, de las cuales la amplia mayoría no han sido legadas (y, en rigor, se discute si siquiera fueron escritas) aumentó la creencia en el supuesto desinterés eckhartiano por la actividad académica.

Sin embargo, en estas últimas décadas se han realizado una serie de estudios al interior de su obra latina que la han revitalizado completamente y que, contrario a lo que se supo creer, la posicionan como un conjunto de textos con coherencia interna, principios identificables, argumentos bien trabajados y, también, con una presencia indiscutida de referencias a textos célebres de la época. Por todo esto, Eckhart ha logrado en las últimas décadas reposicionarse como un autor inscripto en la escolástica de su tiempo. Incluso, esto se condice en mayor medida

1 Actualmente, en líneas generales, ningún estudio puede obviar la necesidad de poner en diálogo las dos obras, la alemana y la latina, para dar cuenta del pensamiento eckhartiano. Con todo, sigue habiendo dos tendencias distintas en las investigaciones respecto a cuál se debe entender como el objetivo teórico del Dominico: si buscó presentar el camino hacia una experiencia mística, o si su producción apuntó a la constitución de un sistema teórico metafísico especulativo. Para textos que elevan el carácter místico, véase Lanzetta, 1992; Sanabria, 2015. Para textos que, más bien, apuntan a elevar el carácter intelectualista de la obra eckhartiana —enfoque que compartimos-, véase: Caputo, 1974; Beierwaltes, 1972, y la bibliografía que se presentará a lo largo de este trabajo.

2 Meister Eckhart (1936a: 148): "videlicet quantum ad generals et sententiosas quasdam propositiones; item quantum ad diversarum quaestionum novas, breves et faciles declarations; adhuc autem tertio quantum ad auctoritatum plurimarum sacri canonis utriusque testament raras expositiones". 
con los descubrimientos biográficos que lo colocan como un activo actor de la orden dominica y, sobre todo, justifica el llamado a ser maestro de Teología en París no solo en una, sino en dos ocasiones (1302/3 y, luego, 1311/12). Eckhart pudo impartir clases en dos períodos en la cátedra de Teología de París, en la que hubiera sido la cátedra de Tomás de Aquino, lo que demuestra que debía ser reconocido en esos momentos como alguien capaz de desplegar una digna función como representante académico dominico. A su vez, esta es otra hipótesis posible para explicar la extensión no tan amplia de su obra latina: no era desinterés, ni tampoco un estar únicamente abocado a la prédica mística, sino que su tiempo lo ocupaba, en gran medida, todo un conjunto de actividades administrativas y de representación de la orden dominica frente a las diversas autoridades de época (Sturlese, 2012).

De las obras presentadas en el Prólogo general, el Prólogo a la obra de las proposiciones es uno de los pocos textos que han perdurado ${ }^{3}$. Este trabajo busca continuar la línea de los estudios que abogan por la identificación de una estructura metafísica definida en los textos latinos de Meister Eckhart. Consideramos, precisamente, que hay una base teórica identificable en el conjunto de su producción, y que la sistematización de ciertos elementos abre a la apreciación de la misma. Este texto, el Prólogo a la obra de las proposiciones, se presenta como uno del cual se pueden extraer las notas necesarias para elaborar un marco conceptual de la estructura del mundo creado.

Recientemente, a propósito de este Prólogo, A. Quero Sánchez publicó un artículo donde discute la hipótesis de que este texto no debería ser entendido como un prólogo, sino más bien como el Tratado sobre el ser, lo que es y la nada al cual el mismo Eckhart hace referencia en otras de sus obras, particularmente en Sermones y lecciones sobre el capitulo 24 del Eclesiástico (Quero Sánchez, 2016) ${ }^{4}$. Esta posibilidad, interesante en sí misma, se condice con la propuesta de entender a este texto como la explicitación de la ordenación metafísica del mundo creado, del momento de Dios salido de sí mismo (ebullatio) ${ }^{5}$.

\footnotetext{
3 Para una lista de las obras legadas véase Bara Bancel, 2015: 101-102.

4 Luego de un amplio estudio comparativo entre manuscritos, se presenta como dato filológico central la posible confusión que un escriba pudo haber tenido entre el término "prooemialiter" - que es el que se encuentra en los manuscritos legados-, con "principaliter", término usualmente utilizado para delimitar las distintas secciones del cuerpo de texto (Quero Sánchez, 2016: 266).

Véase para este esquema el conocido pasaje del Comentario al Éxodo que es uno de los loci más claros para comprender el proceso creacionista según lo plantea Eckhart: "tertio notandum quod repetitio, quod bis ait: sum qui sum, puritatem affirmationis excluso omni negativo ab ipso deo indicat; rursus ipsius esse quandam in se ipsum
} 
Las últimas dataciones tanto de este texto como de la I Cuestión parisiense señalan que fueron escritos en un mismo período de tiempo: la Cuestión entre 1302/3, y el Prólogo, terminado aproximadamente en el 1305, podría incluso haberse comenzado a escribir en los mismos años que la Cuestión. De esta manera, si entendemos a la Cuestión como el estudio de la naturaleza divina de Dios in quantum causa (principalmente eficiente, aunque también hay menciones a su carácter de causa ejemplar), al dar cuenta de su ser-intelligere que opera como fundamento para instituirse, precisamente, en causa de la Creación —en términos del Comentario al Éxodo, la I Cuestión dirime acerca de las notas intrínsecas de Dios-causa tal que en él se da la bullitio, la reflexión interna en y sobre sí mismo-, entonces es razonable suponer que, luego de haber desplegado ciertas notas teóricas de la causa de la Creación en esta Cuestión, se haya adentrado en la escritura del Prólogo/Tratado, para atender, en este texto "de continuación", al efecto de esa causa. Y por ello el Prólogo trata sobre el esse, sobre "la primera de las cosas creadas". De esta manera, este texto se aboca a desandar qué se significa cuando se habla del "esse", cómo se mueve, cómo constituye a las creaturas, si es que las creaturas se pueden diferenciar de este, cómo se relacionan entre ellas: en síntesis, un análisis metafísico de la ordenación del mundo finito.

En lo que sigue, ordenamos la exposición en dos secciones: en primer término, se plantea la hipótesis de lectura donde se presenta la posibilidad de entender al "ser" en tres momentos lógicamente distinguibles -el ser (esse) en sentido absoluto, el ente en abstracto (ente) como representación de la individualidad indeterminada y el ente hoc como la creatura particular-. Luego, en segundo término, se rastrea esta hipótesis en los argumentos esgrimidos en el Prólogo, siguiendo la propia estructura eckhartiana que lo ordena a partir de cuatro proposiciones nucleares. Aquí es que buscaremos señalar cómo las proposiciones que el dominico formula respecto al esse refieren a la mostración de este como "representante conceptual" de la mediación y, así, como la estructura metafísica del mundo creado. Por traslación, la relación entre el esse mismo (todo lo que es, en cuanto que es) y el esse hoc expresa, a la vez, la dialéctica de

reflexivam conversionem et in se ipso mansionem sive fixionem; adhuc autem quandam bullitionem sive parturitionem sui - in se fervens et in se ipso et in se ipsum liquescens et bulliens ... Vita enim quandam dicit exseritionem, qua res in se ipsa intumesens se profundit primo in se toto, quodlibet sui in quodlibet sui, antequam effundat et ebulliat extra" (Meister Eckhart, 1992: 21-22).

6 En la Cuestión discute la naturaleza de la causa y la escinde del esse, por cuanto este es su efecto. Sostiene: "en cuanto llegamos al ser, al punto llegamos a la creatura" (unde statim venimus ad esse, venimus ad creaturam) (Meister Eckhart, 2007: 41). 
identidad y diferencia metafísicas que es constitutiva de la relación entre Dios y las creaturas.

\section{NOTAS PRELIMINARES}

Consideramos que en las pequeñas notas preliminares que Eckhart coloca al comienzo de este texto puede rastrearse el desagregado lógico del "esse" que nos interesa aquí exponer. Según Quero Sánchez, estas notas serían las que, propia y únicamente, el dominico quiso colocar como prólogo a la Obra de las proposiciones. Esto se condice certeramente con lo que aquí planteamos: en el prólogo expuso sucintamente los fundamentos teóricos que, luego, habría de desplegar en los distintos textos que trabajarían "mil proposiciones o más" (Meister Eckhart, 1936a: 148). El primer tratado de ese compendio, el Tratado sobre el ser, lo que es y la nada, se dirige directamente al despliegue minucioso de esos postulados.

En efecto, Eckhart comienza el apartado de las notas preliminares con la siguiente proposición: que "Esse est Deus". El objeto de estudio al que se abocará es, así, el esse. Y, agrega, el esse es Dios. Si volvemos sobre la I Cuestión, en esta se sostiene que Dios, en tanto causa, no es su efecto. Así, este Dios no es Dios-causa, sino Dios-efecto de sí mismo. Este esquema, que recorre la obra de Eckhart y es uno de los distintivos más explícitos de su carácter neoplatónico, puede atenderse en el famoso pasaje del Comentario al Éxodo, donde señala que el proceso de la bullitio interna de Dios -Dios-causa - lleva al derrame de sí, por fuera de sí, a la ebullatio -Dios-efecto- (Meister Eckhart, 1992: 21-22).

"La primera de las cosas creadas es el ser" ". El plano de la ebullatio es el que aquí se despliega. Ahora bien, ¿cómo disponerse hacia este? En las proposiciones siguientes se deja claro que las creaturas - valga la tautología- son, porque son. El ser de las creaturas no es otro distinto a ese primer ser creado. Así, este ser que se presenta como lo primero creado no es una sustancialidad separada de las creaturas, en el cual luego, tal vez, podrían participar, sino que es el ser mismo de estas, su entidad propia. Específicamente, en la tercera proposición que analiza, Eckhart sostiene que la dación del ser es inmediata ${ }^{8}$. Este proceso de la ebullatio, así, no contempla la instauración del "esse" como instancia intermedia

\footnotetext{
7 En palabras de Eckhart: "unde statim venimus ad esse, venimus ad creaturam" (Meister Eckhart, 2007: 41).

8 "et hoc est tertium principale inter quattuor superius praemissa, scilicet quod omne ens et singulum non solum habet, sed et immediate, absque omni prorsus medio, habet a deo tutum esse, totam suam unitatem, veritatem et totam suam bonitatem" (Meister Eckhart, 1936: 172-173). Para un análisis específico de esta, véase la sección dedicada a este tópico de este trabajo más adelante.
} 
entre Dios-causa y las creaturas: las creaturas son ese esse mismo, de manera inmediata.

$\mathrm{Y}$ aquí es que comienza a abrirse la necesidad para la comprensión del "esse" como un concepto abstracto del mundo: como la representación intelectual de la mediación, del efecto en sí mismo. El mundo se encuentra compuesto por creaturas, lo asequible perceptiblemente es el conjunto de las cosas finitas que se mueven en este: los entia haec. Ahora bien, es posible desarrollar un análisis tal que permita apresar aquello que las reúne a todas, aquello que permite que se presentan como cosas en el mundo: el mismo acto de, precisamente, ser. Y la condición de posibilidad para ser-algo-ahí es, sencillamente, ser. Las cosas pueden ser algoque-está-ahí con ciertas características debido al acto de ser. Así, si el ser es condición de posibilidad para las creaturas, entonces el esse se presenta como lógicamente anterior a las creaturas, aunque la constitución de estas sea inmediata. De esta manera, metafísicamente se puede sostener que el esse es la primera de las cosas creadas: es lo primero lógicamente, antes que cualquier particularidad o diferencia, pues es necesario ser (ens), para poder, luego, ser-algo (ens hoc).

A continuación, señalamos cómo es posible descubrir esta distinción en las notas preliminares.

\subsection{Tres "momentos" lógicos del esse}

Para comenzar la exposición de esta proposición ("Esse est deus"), Eckhart señala la importancia de dos notas preliminares. En primer lugar, que "así como 'blanco significa la sola cualidad', según dice el Filósofo [Aristóteles], del mismo modo ente significa el solo ser" [Quod sicut album solam qualitatem signat, ut ait philosophus, sic ens solum esse signat] (Meister Eckhart, 1936b: 166) ${ }^{9}$. Y, en segundo lugar, un par de renglones más abajo: "debe juzgarse de una manera acerca del ente y de otra acerca del ente este o aquel; similarmente, de una manera acerca del ser en sentido absoluto y en cuanto tal, sin ninguna adición, y de otra acerca del ser de este o de aquel" [quod aliter senciendum est de ente et aliter de ente hoc et hoc. Similiter autemdicendum de esse absoluto et simpliciter nullo addito, et aliter de esse buius et buius] (Meister Eckhart, 1936b: 166-167).

En estas notas se encuentran los tres "momentos" lógicos del esse. La primera indica la distinción conceptual que puede abstraerse entre el esse y el ens en lo que respecta a la "efectividad" del ser. El esse, que la segunda nota preliminar llama "esse absolutum", es la pura base existencial del mundo finito. Incluso, es la conceptualización del mundo como tal: la

9 Las citas en español siguen la traducción de D’Amico \& Ludueña (2018). 
estructura fundante de todo lo que es $(e n s)^{10}$. Cuando se lo analiza desde Dios-causa, el esse es la expresión de la mediación, esto es, del efecto, la aparición del plano finito. La distinción conceptual entre el "esse absolutum" y el "ens" señala a las referencias de uno y otro: mientras que el "es$s e$ " se dirige a la pura abstracción, el "ens" es ese mismo esse dado a las creaturas. En efecto, "ens solum esse signat" porque es el puro ser (esse), "nullo addito". Y, con todo, al decir "ens" nos acercamos más a la idea de que el ser se despliega a sí mismo en las creaturas, esto es, en individualidades determinadas ("esto y aquello" [huius et buius]). La distinción conceptual descansa en el hecho de que, aunque ambos plenamente indeterminados, el "ens" contiene en su concepto la individuación, el "corte" del esse absolutum. Este apunta, como concepto, a mantenerse separado de las creaturas. Al contrario, el ens busca señalar el efectivo movimiento del esse en las creaturas. El esse como concepto se mueve más allá de cualquier efectividad, mientras que el ens la tiene en sí mismo. Esta opera clara y únicamente como una distinción lógica, y busca elevar el concepto del esse y aislarlo de cualquier entidad para clarificar el orden metafísico que Eckhart está presentando.

Ahora, la segunda nota introduce a las creaturas - a la individualidad, a la determinación particular-. Al interior del mundo creado, este "todo lo que es" puede entenderse como una colección de entia: la fórmula "todo lo que es, in quantum es", aunque plenamente indeterminada, contiene en sí la referencia a la diferencia. El "todo" —omnia - es la representación de un conjunto. Sin embargo, no hay ninguna indicación de alguna suerte de particularidad definida y, por lo tanto, la categoría de "eso (ente) que es (ens)" - así como la de "todo lo que es, in quantum es" (esse absolutum) - se mueve en la esfera de la indeterminación. "Eso que es", sin embargo, ya refleja un indicio que muestra cómo va a constituirse este mismo en algo definido (ente hoc).

$Y$, de esta manera, dice "debe juzgarse de una manera acerca del ente y de otra acerca del ente este o aquel". El "ser-individualizado" es el "ente" como "concepto-de-esto-que-es". En efecto, el ente mismo es la manifestación de la base metafísica que lleva a la constitución de "algo" —“algo" como "ente-determinado"- pero que es, en sí mismo, comple-

10 Mayer llama al "esse absolutum", "the common act to be" [el acto común de ser]: "God causes the act to be of the whole universe, which has the power to actualize everything, including every form. In this sense, Eckhart also says: the providence of God -in every operation concerning creatures and in the creation of the universe itself - regards primarily and through itself the common act to be and good and the act to be and good of all (respicit esse et bonum omnium et commune)" (Mayer, 2017: 532). En los términos que venimos utilizando: Dios-causa, esto es, Dios en tanto causa primera, crea "el acto de ser de todo el universo", esto es, al esse, Dios como efecto. 
tamente indeterminado: es la "imagen mental" de "esto que es", es el concepto de un sustrato-todavía-no-determinado. Por su parte, el "ente hoc" es ya "eso que es esto determinado". Allí descansa la diferencia conceptual entre uno y otro.

El mismo movimiento cognitivo sucede con la distinción entre el "esse absolutum" y el "esse hoc": "[debe juzgarse] de una manera acerca del ser en sentido absoluto y en cuanto tal, sin ninguna adición, y de otra acerca del ser de este o de aquel". Cuando reflexionamos acerca del "puro" ser, entonces allí tratamos de conceptualizar la "pura indeterminación absoluta", el fundamento de todo lo que es, el concepto de la multiplicidad del mundo (que, como tal, es en sí mismo uno). Y debemos entender que ese esse absolutum es el que constituye a las creaturas determinadas. Y, así, es el mismo ser que el "ser de esto o aquello". Sin embargo, precisamente porque este último está ya determinado, opera en un nivel de análisis diferente.

En rigor, el "ser de esto o aquello" es aquel que es aprehensible de manera más directa por el conocimiento humano. Cuando vemos una cosa $x$, la identificamos como "algo que estáles ahí en este mundo". Sin embargo, un proceso de abstracción ulterior lleva al desagregado de esta cosa tal que podemos conceptualizarla como la "estructura vacía" que es el "ente". El ente es "eso (ente) que es (ens)". Y, finalmente, ese "ens" se "mueve" hacia el puro "esse absolutum", que es el fundamento subyacente de la posibilidad de ser de las creaturas.

\subsection{Análisis lógico-predicamental}

Este mismo marco metafísico antes expuesto puede descubrirse en clave predicamental: si se busca dar una definición, plantear "esto es" no es suficiente en vistas de alcanzar lo que se quiere denotar. En rigor, por lo que expone Eckhart, el "esto" incluye ya en sí el "es", puesto que la identificación de un "esto" señala, en efecto, que hay un sustrato existente.

El "esto" (ens) es Dios in quantum existencia (esse absolutum) dispuesto a definirse. El "esto" puede instituirse puesto que el "esse est Deus", esto es, debido a la ebullición divina que resulta en la constitución del mundo. En términos del Comentario al Éxodo, al ebullir, Dios abre al reino de la multiplicidad y la aparición de los estos y aquellos. Ahora bien, en tanto es Dios mismo quien sale de si mismo, entonces este mismo, in quantum esse, 
es, también, el fundamento de todo lo que es, puesto que es esto mis$\mathrm{mo}^{11}$.

Con todo, sostener que la estructura del mundo es la existencia, esto es, Dios-efecto, no es útil en vistas de la practicidad de la identificación de particularidades definidas. Y allí es donde se presenta el ente hoc. Aunque todas las creaturas son Dios, sin embargo, son distintas unas de otras. Cada creatura se determina al participar en una causa segunda. Esto está ejemplificado por el mismo Eckhart en clave lógica en el despliegue que él hace de la segunda nota preliminar antes mencionada:

Cuando digo que algo es, o predico "uno", "verdadero", "bueno" no solo los cuatro términos mencionados caen en la función de predicado en cuanto segundo adyacente sino que también son tomados formal y sustantivamente. En cambio, cuando digo que algo es esto -por ejemplo, piedra-y que es una piedra, verdadera piedra o esto bueno, los cuatro términos mencionados son tomados en cuanto terceros adyacentes de la proposición y no son predicados sino son cópula o adyacente del predicado ${ }^{12}$.

Predicar "esto es" (cum dico aliquid esse), por sí solo, expresa una estructura constitutiva: el hecho mismo de estar en la existencia. Podría aquí señalarse que, en tanto se sostiene que “aliquid” es, se observa ya la estructura del "ente" como individuo ya diferenciado de otros en el mundo. Ahora bien, este nivel del "ente", como se dijo, posee un grado de indeterminación demasiado amplio. Pues solo establece que, debido al hecho de ser en el mundo finito, en el reino de la multiplicidad, las creaturas necesariamente son determinadas -esto, es decir, al ser esta creatura, no es todo el resto de las creaturas que podría ser. Así, no es suficiente en vistas de la identificación de un ente señalar que "un ente es", más bien, lo se que requiere es señalar las propiedades que construyen esa determinación del ente y que lo separan de todo el resto de lo que podría ser. Por tanto, la definición se completa cuando se dice que "esto es una piedra". Al puro concepto abstracto de "substancia en potencia", a la pura estructura existente, se le inscribe la determinación, y el puro esto se constituye en un algo determinado.

11 Aquí es importante no olvidar lo que fue dicho al comienzo: que esta effundo, aunque muestra la continuidad ontológica entre la causa y el efecto, constituye una mediación real y efectiva, tal que la causa y el efecto son dos esferas diferentes.

12 "Cum enim dico aliquid esse, aut unum, verum seu bonum predico et in predicato cadunt tamquan secundum adiacens, premissa et formaliter accipiuntur et substantiue. Cum vero dico aliquid esse hoc, puta lapidem, et esse unum lapidem, uerum lapidem, aut bonum hoc, scilicet lapidem, premissa accipiuntur ut tercium adiacens propositionis, nec sunt predicata, sed copula uel adiacens predicati” (Meister Eckhart, 1936b: 167). 
Aquí, entonces, se vuelven a observar los tres "momentos": Dios se encuentra realmente en todas las creaturas, como se observa en el plano proposicional en el hecho de la predicación: el "aliquid esse" expresa la existencia, y esa existencia es Dios. Además, sostener que "algo es", por sí solo, expresa la abstracción de una cosa, esto es, de una "entidad (ente) que es (ens)". Pero para dar cuenta realmente de aquello que se despliega en el mundo, hay que agregarle la determinación: "aliquid est lapis". En vistas del objetivo central que creemos posee este Prólogo, lo que vale rescatar principalmente es que, sencillamente, sin la existencia, el esto se desvanece. Así entonces, sin Dios, las creaturas son nada, como se explayará de forma explícita sobre esto más adelante ${ }^{13}$.

¿Qué busca, entonces, expresar el dominico? Que la condición necesaria para que una entidad entendida como particularidad se concretice es el existir en el mundo: la aparición del esse es anterior lógicamente a la constitución en un esto determinado (ente hoc et hoc). Aquí opera el juego entre la causa primera y las causas segundas: la primera es principio efectivo de todo lo que es, y su dación del ser es metafísicamente anterior a la acción de las causas segundas, que se instituyen como principios formales, los que determinan a esas entidades que ya se inscribieron en el mundo debido a su ser dado ${ }^{14}$.

Recapitulando, hay, así, un movimiento abstractivo que comienza en el ente hoc, que lleva al ente y que se abre al esse absolutum. La atención que se dispone sobre uno u otro estadio responde, en gran medida, al objeto de estudio pretendido y al método utilizado: si el análisis es inductivo y parte de aquello dado en el mundo, entonces se comienza con la creatura. Esta se presenta como un ente hoc que se puede delimitar y abstraer conceptualmente hasta llegar a su calidad de puro ente vacío de toda determinación, como una suerte de plenitud dispuesta a particularizarse ${ }^{15}$, pero que no es el puro esse pleno, sino una suerte de sustrato ya vitalizado que debe instituirse en algo particular. Luego, si se opta por un análisis deductivo que comienza en el fundamento del mundo, entonces se busca apresar, precisamente, a ese puro esse absoluto que es fundamento de la existencia de ese ente, y que incluso es lógicamente anterior a la conceptualización de la individualización de cada creatura particular que refleja

13 "Praeter esse et sine esse omnia sunt nihil, etiam facta" (Meister Eckhart, 1936b: 178).

14 Un locus argumental donde Eckhart presenta y despliega esta dicotomía entre el principio existencial y el principio formal, coincidiendo con la primacía lógica del esse en el análisis de la estructura metafísica del mundo finito es en Meister Eckhart (1994: 50$53)$.

15 La nota de "plenitud" se entiende si, precisamente, lo vemos como ese puro estadio del esse absolutum "delimitado ya en entidades que no se les ha asignado una forma". 
el "ente". Este esse absoluto es la conceptualización del efecto de Dioscausa".

Esto es, lógicamente - pero solo de esta manera- es posible concebir al primer efecto creado, el esse absolutum, como fundamento metafísico separado de sus creaturas. Lo que quiere señalar es, entonces, que el ente "ocurre" en el esse - los entes se mueven en la existencia-, y que este es "base estructural" del ente: la existencia (esse) es la condición de posibilidad para que un ente (ente) sea (ens) y, aun más, para que un ente determinado (ente hoc) sea.

\subsection{El esse como límite conceptual}

Por todo esto, creemos estar en condiciones de sostener lo que se deja entrever en la terminología de la exposición precedente: el esse absolutum es la conceptualización intelectual del orden metafísico de la Creación. Dios-efecto en tanto mundo, el mundo como esse absolutum, la estructura de lo que es, la mediación que hace su aparición en el movimiento de la Creación. Este es la condición de posibilidad para que las creaturas sean y, así, se presenta como lógicamente anterior a ellas, aunque la donación del ser sea inmediata. Lo que Eckhart desplegará a lo largo de este texto es la prioridad lógica de la donación de ser para la constitución del mundo creatural. El esse absolutum es, así, "la primera de las cosas creadas", el concepto del mundo y, así, el concepto de la ordenación de la realidad toda, Dios-causa que crea a su efecto: al ser, al mundo finito.

Esta caracterización de los tres "momentos" del esse, como recién se señaló, responde a un análisis conceptual propio de la actividad abstractiva del entendimiento humano. Esto no solo se advierte si observamos la proposición antes mencionada —aquella que sostiene que la dación de ser es inmediata-, sino que Eckhart mismo lo expone explícitamente en el Comentario al Génesis, cuando señala que la creación es un movimiento eterno: la separación en "momentos" responde a la necesidad humana de operar a través de proposiciones sucesivas, pero no al carácter real de la Creación:

16 Esto se encuentra también presente en palabras de Mayer: "the phrase 'things have the act to be' refers to participation. The term 'being' (ens) connotes something participating in the act to be (esse). Thus, 'being' names the act to be in a concrete way (in concreto) whereas 'act to be' signifies it in an abstract way (in abstracto). Eckhart writes: being signifies the act to be though it cosignifies or connotes also that, which is", Mayer, 2017: 516. El "esse" es la existencia sin más, aquella que permite instituir a los "entia" como constructo dispuesto a individualizarse. Lo concreto del "ens" es que es el concepto de "eso que es-particular". Y, finalmente, el "ens hoc" es un ente ya determinado. 
El "principio" en el que "Dios creó el cielo y la tierra" es el primer-simpleahora de la Eternidad ... Las cosas que Dios hace al mismo tiempo no pueden ser expresadas por nosotros "de una sola vez". Esto se debe a que, a diferencia de nosotros, el hablar de Dios es su hacer, y, también a diferencia de nosotros, su hablar es la causa de todo su obrar y de todas sus partes ${ }^{17}$.

En lo que sigue, señalaremos la aparición de esta conceptualización al interior del texto, que, según Quero Sánchez, sería un tratado en sí mismo, dispuesto a desplegar estas notas preliminares que presentan la distinción categorial eckhartiana.

\section{PROPOSICIONES ECKHARTIANAS}

$\mathrm{Al}$ interior del texto mismo hay un ida y vuelta constante en el uso del término "Deus". En lo que sigue, nos interesa la referencia a Diosefecto, esto es, a Dios como esse, como "la primera de las cosas creadas". Con todo, precisamente porque se discute sobre el esse creado, las menciones a Dios-creador son constantes. El uso del mismo término responde a la estructura de la realidad toda que antes mencionamos sucintamente. Dios "en toda su expresión", Dios como "divinidad toda", Dios "superabundante" es la realidad toda que se mueve al interior de sí. En ese movimiento se vislumbra la mediación de la causa y el efecto, la causa primera y el mundo finito. Sin embargo, en tanto ambos son "momentos" de este Dios "en toda su expresión", reciben ambos el nombre "Deus". Como se ve, la identificación de la causa primera como "Dios" es el uso tradicional, y, así, lo interesante es el uso del vocablo para referir al mundo finito, como plexo de existencia. Este uso viene a reforzar la estructura de la realidad toda como una gran unidad que se mueve en sí, por un lado, y también señalar el proceso de ebullición de Dios-causa que lleva a la institución del esse, por otro. Explicitar que el esse es Dios es remarcar este tipo de proceso creacionista. Este juego de identidad y diferencia es constitutivo de la filosofía neoplatónica, que debe marcar las mediaciones ordenadoras de la realidad (Dios-causa y el mundo finito), al tiempo que debe reunirnos (el mundo finito es efecto ebullido de Dios, y ambos, causa y efecto, se mueven en una unidad superadora).

17 "principium, in quo deus creavit caelum et terram, est primum nunc simplex aeternitatis (n. 7) ... quae deus simul facit, non possunt simul a nobis dice, tum quia dei dicere est suum facere -secus in nobis- tum quia dei dicere est causa totius sui operis et omnium partium -secus in nobis- (n. 8)" (Meister Eckhart, 2015: 190-191). 
En lo que sigue, intentaremos aclarar en la mejor medida de las posibilidades este juego terminológico constante, y señalar, en cada caso correspondiente, cuándo "Dios" refleja a la causa primera, y cuándo al "esse" como efecto de Dios-causa.

2.1. "Dios solo es en sentido propio lo que es (ens), lo uno, lo verdadero y lo bueno"

"Quod solus deus proprie est ens, patet Exodi 3: ego sum qui sum" (Meister Eckhart, 1936b: 168) ${ }^{18}$. Luego realiza la identificación entre la unidad y Dios, al señalar que tanto Proclo como el Liber de causis plantean a Dios como lo uno y la unidad y, finalmente, cierra este párrafo reuniendo ambos conceptos al expresar que:

El término "uno" significa la negación de la negación. Por esta razón solo compete al ser primero y pleno, cual es Dios, de quien nada puede ser negado porque pre-contiene e incluye de manera simultánea a todo $\operatorname{ser}^{19}$.

Esta frase sostiene y llama a entender la identificación de Dios y el ser. ¿Cómo se da esta identificación? En que solo este es "primero y pleno esse". Y de este nada puede ser negado, pues "precontiene e incluye" a todo lo que es. Así como en el marco metafísico general trabajado en la I Cuestión el esse se presenta como "primer efecto" y, así, como negación de Dios in quantum causa, aquí en este Prólogo se discute acerca del movimiento de este esse efectuado que es Dios superabundante ${ }^{20}$, pero

18 En el Comentario al Éxodo, Eckhart hace uso de la fórmula "ego sum qui sum" para expresar la supra-afirmación divina de Dios entendido como principio de la realidad toda, siendo la misma realidad toda. No es aquí el caso, donde lo está identificando con el esse absolutum que, en rigor, es, al interior de la Divinidad supra-abundante, un momento de esta. Sin embargo, no hay aquí confusión terminológica ninguna, pues la riqueza de esta fórmula, así como la de la cláusula "negatio negationis" es que se puede usar indistintamente para expresar diversas relaciones al interior de la unidad divina en toda su expresión. En este caso, en efecto, el tratamiento que le otorga a "ego sum qui sum" no refleja la repetición del verbo como supra-afirmación sino la relación entre el esse absolutum y el esse hoc, y cómo el primero es fundamento del segundo.

19 "Preterea li unum est negatio negationis, propter quod soli primo et pleno esse, quale est deus, competit, de quo nichil negari potest, eo quod omne esse simul prehabeat et includat" (Meister Eckhart, 1936b: 169).

$20 \mathrm{Si}$, siguiendo a la escuela neoplatónica, la realidad toda es Dios, entonces, Dios se puede decir de muchas maneras: Dios "superabundante" refleja la unicidad absoluta de la realidad en su conjunto. A su vez, se puede, al interior de esta unidad, identificar como uno de sus momentos a Dios-causa - lo trabajado en la I Cuestión - y, como otro, a Dios-efecto, que es lo que aquí se trabaja en el Prólogo: Dios como el ser creado. Diosefecto no es Dios-causa, y Dios-causa no es Dios-efecto y, con todo, ambos momentos 
ahora, en este plano de discusión, in quantum efecto. En este texto, el esse se presenta como plenitud. ¿Por qué? Porque ya no se está discutiendo la relación entre la causa (Dios causa eficiente) y el efecto (Dios efectuado por sî) que se da en la I Cuestión, sino el movimiento al interior de lo efectuado, el reino de la multiplicidad: y el esse absolutum se erige como primus y plenus, y, así, es este el que niega la negación por instituirse como unidad absoluta en este plano de discusión. ¿Cuáles son las exponentes, entonces, aquí, de la negación? Las creaturas, por su carácter determinado.

Y lo que aquí señala Eckhart es que el esse absolutum pre-contiene a todos los esse hoc. ¿Por qué? Precisamente porque la existencia de las creaturas es $s u$ existencia. Todo lo que es determinado se encuentra en Él, pues Él es lo que es, en tanto que es, esto es, todo lo existente (cada ente), es (esse). Por ello Eckhart plantea como primer apartado que "Dios solo es en sentido propio lo que es (ens)". Aquí podemos recordar lo que sostuvimos en el apartado anterior: que la única distinción entre el "esse" y el "ens" descansa en los niveles de abstracción en los que se mueven. Y, precisamente, plantear que "solo Dios (esse) es lo que es (ens)" muestra este movimiento desde la pura abstracción de la "existencia como tal" (Dios/esse) a la existencia efectivamente desplegada en el mundo, en "lo que es" (ens). Ambos refieren, como se dijo antes, a la pura indeterminación del ser. Así, decir que Dios es "lo que es (ens)" concuerda con nuestra interpretación que afirma que Dios-efecto es la pura estructura absoluta del mundo, tal que Él es todo lo que es. Luego, Él es condición de posibilidad para la existencia de las cosas. Y, con todo, como fue dicho, no es perceptible sino ya como ser de las creaturas individuales.

2.2. "A partir de Él todas las cosas son, son un uno, son verdaderas y son buenas"

Como se vio, en el primer punto de los cuatro que estructuran el texto, Eckhart atiende a la distinción entre el esse absolutum y el esse hoc et hoc. Esta le permite desplegar el orden metafísico que señala que el esse es la base subyacente de todo lo que es y, así, es eso que es. Esto lo resume sucintamente cuando dice "¿de qué modo algo existiría sino desde el ser o sería uno sino desde lo uno?"21.

constituyen a Dios superabundante. Además, este Dios-efecto ebulle desde Dios-causa, señalando una continuación ontológica que, con todo, marca su límite: precisamente, que Dios-causa no es el esse, pues este es "la primera de las cosas creadas", trazando la distinción en el orden de la realidad.

21 "quomodo enim quippiam esset, nisi ab esse et per esse?" (Meister Eckhart, 1936b: 170). 
Luego se enfoca, primariamente, en la constitución creatural como tal. Este pasaje argumental desde el análisis metafísico de Dios-esse, como fundamento subyacente in quantum "existencia de lo que existe", hacia la constitución creatural in concreto, tiene como objeto discutir el fundamento "último" de la presencia en el mundo de las creaturas: más allá que lo característico en vistas de la identificación particular de cada ente este o aquel son sus determinaciones, con todo, la condición de posibilidad para que una creatura se particularice es que esta debe en primer término, sencillamente, ser.

Para exponer esto comienza volviendo a señalar la identidad entre los trascendentales que son unum, verum y bonum. Estos, dice el dominico, "son primeros en las cosas y comunes a todas". Y esto se debe a que "son inherentes solo por la causa primera y universal de todas las cosas". Dios-causa eficiente crea el mundo, esto es, al esse absolutum/en general. Como dijimos, la distinción terminológica entre "esse" y "ens" se puede asentar entre el grado de abstracción máxima que significa el "esse" en tanto pura existencia, por un lado, y el "ens" como mostración ya de "eso que es" instanciado -aunque no particularizado-. Aquí Eckhart, en sintonía con la teoría de los trascendentales, señala que, entonces, el "ens" se presenta siempre unido a lo uno, lo verdadero y lo bueno. Y que, así, al haber una identidad real entre este y los otros trascendentales, entrar a la existencia es ser necesariamente siendo uno, verdadero y bueno ${ }^{22}$.

A continuación, introduce las causas segundas. La exposición encierra un doble propósito: en primer término, señalar la distinción conceptual entre la causa eficiente y la causa ejemplar/esencial y, en segundo término, que lógicamente la determinación requiere previamente para su institución que algo sea. Estas dos notas se resumen en esta frase: "Las causas segundas no quedan excluidas de las influencias de la causa prime$\mathrm{ra}^{\prime 23}$.

Como señalamos antes, en este estadio de la argumentación le interesa señalar a Eckhart que, en la constitución de la creatura, la existencia es lógicamente anterior a la esencia: solo se puede determinar, esto es, limitar, algo que ya está puesto en la existencia. Y precisamente porque esta existencia es plena (esse absolutum) es que las causas segundas pueden influir sobre un mundo ya existente dispuesto a determinarse.

Esta idea, el hecho de que la esencia no puede moverse sino a través de una entidad existente, se expresa de la siguiente manera en el texto:

\footnotetext{
22 Para un desarrollo pormenorizado de la teoría de los trascendentales en Eckhart, véase Tsopurashvili (2012).

23 "Non tamen per hoc excluduntur cause secundarie a suis influentiis [de la causa prima]" (Meister Eckhart, 1936b: 171).
} 
La forma del fuego no da al fuego el ser, sino el ser esto, ni el ser uno, si el ser esto uno - por ejemplo, el ser fuego-24.

En términos que viene manejando en este Prólogo: las causas segundas otorgan el hoc, pero no el ens. Y, remarca el dominico, solo porque el ens se encuentra ya allí es que el hoc puede instituirse.

2.3. "A partir de Él -de manera inmediata- todas las cosas tienen el hecho de que son, de que son un uno, de que son verdaderas, de que son buenas"

En el punto siguiente agrega la noción de inmediatez. Esto no estaba completamente explicitado en lo anterior, pero es fácilmente detectable, y, a su vez, permite fundamentar uno de los postulados más conocidos de Eckhart - y que fue una de sus proposiciones que fue condenada-, a saber, que la creatura, por sí misma, no es nada.

Agrega ahora el dominico que esta dación de existencia es inmediata. Esto significa que:

Todo ente no solamente tiene todo el ser a partir de Dios ... además lo tiene de manera inmediata, sin ningún intermediario ${ }^{25}$.

Entre cada ente (que es [ens]) y el esse no hay alguna Otredad operante. Puesto que, si lo hubiera, entonces esta otredad estaría fuera, "como a

24 "forma enim ignis non dat igni esse, sed esse hoc, nec esse unum, sed ese unum hoc, puta ignem et unum ignem" (Meister Eckhart, 1936b: 171-172). Una exposición similar es la que da en la I Cuestión, cuando señala que "hombre" (como signo de existencia, de sustrato) no deriva de "racional" (como signo de la esencia), sino que "racional" deriva de "hombre". En esta parte del texto utiliza, también, el ejemplo del fuego, y dice: "si el fuego por su propia forma lo pudiese todo, no sólo ser sino también calentar en la forma del fuego, por cuya virtud tendría tal poder, no habría adición o composición alguna". Esto es, si fuese el caso, las creaturas no serían entidades compuestas, sino que serían una unidad equiparable a la divina donde, en efecto, esencia y existencia son una identidad indivisible. Sin embargo, las creaturas son creadas y, como tales, inscriptas necesariamente en la multiplicidad (Meister Eckhart, 2007: 39).

25 "quod omne ens et singulum non solum habet, sed et immediate absque omni prorsus medio habet a deo totum esse, totam suam unitatem, veritatem et totam suam bonitatem" (Meister Eckhart, 1936b: 172-173). Esto está tal cual explicitado en el Prólogo general: "Por lo cual el ser de todo es inmediato a la causa primera y a la causa universal de todo. Por tanto, a partir del ser mismo, 'y por medio de él y en él todo es', no siendo él a partir de otro. Pues lo otro que hay aparte del ser, o no es o es nada" ["propter quod esse omnium est inmediate a causa prima et a causa universali omnium. $\mathrm{Ab}$ ipso igitur esse et per ipsum et in ipso sunt omnia, ipsum non in aliquo nec ab aliquo. Quod enim aliud est ab esse, nichil est" (Meister Eckhart, 1936a: 153). 
un costado" del ser. La justificación eckhartiana para la refutación de esta posibilidad es tan sencilla como coherente, puesto que se limita a decir que: Esse autem est deus.

Si hubiera un algo intermedio, entonces necesariamente, por definición, ese algo sería. Sin embargo, "ser" significa "ser en el ser". La única manera de ser es ser (ens) en el ser (esse): algo solo es en el ser. Entonces, solo lo que está en Dios puede ser. Por tanto, no puede haber un intermediario entre "lo que es" y el ser, porque nada puede ser fuera del $\operatorname{ser}^{26}$.

2.4. 'Los términos 'esto' y 'eso' no agregan ni adicionan nada en absoluto de entidad, de unidad, de verdad o de bondad sobre lo que es (ens) ente"

Eckhart ya había señalado que todo lo que está fuera del ser (Dios) es nada: "todo lo que en una cosa cualquiera no es alcanzado ni penetrado y formado inmediatamente por el ser mismo, nada es" ${ }^{27}$. Como se vio, la noción de inmediatez está íntimamente ligada con la de simultaneidad: cuando Dios-causa da el ser, lo da en toda su extensión. Una cosa no puede ser y no ser. O se está en la existencia, o no se está. Esta dicotomía metafísica entre el ser y la nada es la que está señalando aquí. Por ello habla de "carecer o alejarse": quien carece o se aleja del ser, no es y nada es.

Este argumento le permite sostener que:

A causa de esto, del ente mismo (entitatis universaliter), Dios, nada puede ser negado sino la negación de la negación de todo ser ... Por esto es que lo uno, en cuanto negación de la negación, se relaciona con el ente inmediatísimamente ${ }^{28}$.

Dios, como efecto, como esse pleno, es el puro ser, el ser trascendente ${ }^{29}$. $\mathrm{El}$ esse in abstracto, o, como en esta misma cita lo nombra "entitatis universaliter", que refuerza esta lectura del "esse" como concepto abstracto que refleja la base estructurante del mundo. Este, por su condición de absoluto, no acepta limitación ninguna, no contempla en su constitución la

\footnotetext{
26 Véase Meister Eckhart (1992: 34): "Dios es existencia y él solo da existencia inmediatamente a todo" (Deus autem esse est et solus dat esse immediate omnibus").

27 "Quidquid enim rei cuiuslibet ab ipso esse immediate non attingitur nec penetrator et formatur, nihil est" (Meister Eckhart, 1936b: 173).

28 "Nihil ergo entitatis universaliter negari potest ipsi enti nisi negatio negationis omnis esse ... Hinc est quod unum, utpote negationis negatio, immediatissime se habet ad ens" (Meister Eckhart, 1936b: 175).

29 Para Dios como "ser trascendente", véase: Mojsisch, 2001.
} 
posibilidad de la división. Esto lo justifica amparándose en la noción de inmediatez: el ser se da siempre en toda su plenitud-una. Por tanto, Dios, en tanto esse absolutum, niega la división. ¿Dónde descansa este límite? En los términos esto y eso. Pero el esse absolutum es uno, por tanto, excluye de sí la determinación. Entonces, el esse absolutum niega la negación. Y por eso se da todo él mismo inmediatamente a cada ente constituido ${ }^{30}$. Esto explícitamente lo señala cuando dice que: "se relaciona lo uno con todo lo que es uno cualquiera sea el modo o la diferencia de lo uno"31. Las causas segundas instituirán a cada ente como "este ente" o "aquel ente", y, luego, a su vez, cada uno será un ente individual. Sin embargo, todos comparten por igual el hecho mismo de, justamente, ser entes: de estar en la existencia. Ese es el esse absolutum.

Y por ello el "esto" o "aquello" no agrega nada. Señalar esto, dice Eckhart, permite establecer el esse absoluto de Dios-efecto. Expone esto de dos maneras: primero, con ejemplos - materia y forma; partes y su todo; hombre asumido por el Verbo-; luego, por medio de razones. Los ejemplos tienen el objeto de negar que, en este caso, la determinación pueda donar existencia. Para ello expone compuestos donde señala cuál es la finalidad de cada "polo" constitutivo y se encarga de exponer cómo se identifica en un compuesto la función de cada cual, y cómo una parecería ser un principio más fundamental para el compuesto que otra.

Con todo, es interesante que, a la vez, eleva la importancia de la determinación, pues en los ejemplos remarca que el hecho de no ser el principio del compuesto no quita que no cumpla un rol necesario. Así, en el caso de la determinación, esta puede no dar ser, pero esto no quita que su presencia en el compuesto sea necesaria. Sin embargo, aunque necesaria, no se ocupa de dar el ser, que es lo que le interesa remarcar aquí.

Materia y forma. Sostiene Eckhart aquí que "la materia no aporta ser alguno al compuesto, ni tiene por sí ningún ser por fuera del ser mismo

30 Esto es lo que permite todos los tratamientos propiamente neoplatónicos respecto a que cada creatura encierra a la creación. Pues su existir mismo responde al puro ser absolutamente uno. Las causas segundas determinan al puro ser, esto es, lo inscriben como una particularidad definida, como una entidad finita inscripta en la multiplicidad. Dentro de estas creaturas, el ser humano, como creatura predilecta de Dios, puede volverse sobre sí mismo para descubrir su ser Dios, que no es más que ser todas las creaturas en una unidad indisoluble.

31 "Et sicut se habet de ente ad entia, sic se habet de uno ad omne quod unum est quocumque modo sive differentia unius" (Meister Eckhart, 1936b: 175-176). 
que la forma da al compuesto". ${ }^{32}$ En este paralelismo, la materia ocupa el lugar del "esto o aquello". Y, expone el dominico, aunque no aporte ser, con todo, "no decimos que la materia es nada", porque, en efecto, es constitutivo del compuesto que es la sustancia". Así, aunque "no agregue ser", con todo participa en la unidad que es la sustancia. De la misma manera, aunque la determinación no agregue ser a la entidad, con todo es constitutiva de la misma.

Partes y su todo. "Ninguna de cada una de las partes aporta ser alguno a su todo, sino que más bien ellas reciben todo su ser de su todo y en su todo" 34 . Esto hace recordar lo que expuso antes respecto a las causas segundas, que reciben el influjo divino que les permite concretizarse y desplegarse en la entidad existente. Así, estas son en virtud de provenir de la causa primera, y, en rigor, de "inscribirse" en algo que es animado por el ser de Dios. Y, con todo, estas son operativas en la constitución del compuesto que es la creatura.

Hombre asumido por el Verbo. Aquí expresa el momento de la encarnación divina. Y señala que en Cristo admitimos "un único ser personal hipostático del Verbo mismo" y, de todas maneras, se sostiene que Cristo fue un hombre entre los hombres ${ }^{35}$. El hecho de que Cristo fue un hombre individual no hizo disminuir en absoluto su carácter divino. Y, agrega Eckhart, incluso más, fue constitutivo de esa divinidad encarnada. Entonces, este ejemplo refleja el núcleo de lo que quiso señalar: que así como el ser-ente de Cristo -la limitación propia de la creatura- no agregó ni sumó nada a la esencia divina, a la vez fue un elemento necesario de ese mismo Verbo que buscaba encarnarse. De la misma manera, la determinación -el hoc- "no adiciona ser" pero tampoco debe entenderse como una limitación "contra-producente" sino, más bien, necesaria para la constitución de los compuestos que son las creaturas, propias del movimiento divino ${ }^{36}$.

32 "Constat quod materia nullum esse affert composito nec habet ex se esse aliquod penitus praeter esse idem, quod dat forma composito" (Meister Eckhart, 1936b: 176).

33 "Nec tamen propter hoc dicimus materiam esse nihil, sed substantiam et partem alteram compositi” (Meister Eckhart, 1936b: 176).

34 "Partes singulae nullum esse proprsus afferunt suo toti, sed potius totum suum esse accipiunt a suo toto et in suo toto" (Meister Eckhart, 1936b: 176-177).

35 "In homine assumpto a verbo concedimus unicum esse personale hypostaticum ipsius verbi, et nihilominus Christus vere fuit homo univoce cum aliis hominibus" (Meister Eckhart, 1936b: 177).

36 En efecto, la propuesta eckhartiana busca constantemente expresar los momentos al interior de la divinidad. Y la ebullición divina, y la consecutiva construcción de la multiplicidad que es el mundo finito, son estadios necesarios de ese Dios supraabundante: sostener un sistema de índole neoplatónico no excluye en absoluto la posibilidad de un estudio detenido del amplísimo abanico que comprende el mundo creatural y de 
Luego lo expresa por razones. El primer argumento refuerza que solo la causa primera (Dios in quantum causa) dona ser: solo la causa primera dona el ser, esto es, constituye a través de su operación al esse absolutum. Por tanto, "ningún esto o aquello da el ser", pues solo Dios lo ha$\mathrm{ce}^{37}$.

El segundo argumento se relaciona directamente con el anterior. Ahora ya no se trata de que el "esto o aquello" no es causa primera y, entonces, no puede donar el ser, sino que sostiene que, en el caso que pudiera hacerlo, entonces Dios-causa no sería pleno por sí, como dice el Liber de causis, puesto que habría otro que da el $\operatorname{ser}^{38}$. Esto se liga, además, con lo que ya señaló en el punto anterior respecto a la inmediatez de la dación divina y la imposibilidad de que haya algún otro intermediario. Puesto que, si el "esto o aquello" pudiera donar el ser, entonces estaría por fuera de Dios. Sin embargo, eso implicaría que esté por fuera del ser y, así, sería nada. Pero la determinación es (en efecto, la exposición eckhartiana señala constantemente la fórmula "ens hoc et hoc"), y, así, no es nada. Por tanto, es imposible que esté por fuera del ser.

El tercer argumento ${ }^{39}$ sigue, en la misma línea, negando que la creatura pueda tener por sí misma esse. La comparación presentada puede en un primer lugar confundir, por cuanto parecería estar presentando una analogía basada en la relación sustancia/accidente. Sin embargo, los términos operan como analogía para lo que sería el polo del "esse" (la blancura), por un lado, y el polo de la "creatura" (el escudo), por otro.

Un escudo blanco, en cuanto blanco, recibe todo su ser blanco a través de la blancura y, por sí mismo, ni tiene un algo de blancura en absoluto ni tampoco refluye nada -en cuanto escudo- hacia la blancura misma ${ }^{40}$.

Así, si realizamos un proceso de abstracción intelectual podemos llegar a conceptualizar a tal creatura $x$ como "esto-determinado", en este caso, un escudo y, con todo, el concepto-escudo por sí mismo -y, así,

otorgarle a este la atención que merece, puesto que su estructura responde al movimiento divino.

37 "Omne dans esse creat et est causa prima et universalis omnium ... Igitur, nihil hoc aut hoc dat esse" (Meister Eckhart, 1936b: 178).

38 “Primum est dives per se', ut in De causis dicitur, sed nec esset 'dives per se', sed nec 'primum', si quid aliud daret esse praeter ipsum” (Meister Eckhart, 1936b: 178).

39 Estructuralmente, Eckhart coloca como tercer argumento el que "las creaturas por sí mismas son nada" pero, en vistas de la consecución explicativa, es mejor dejarlo para el cierre de la argumentación.

40 "Scutum enim album totum suum esse album, inquantum album, accipit per albedinem, nec quidquam prorsus albedinis ex se habet nec refundit, inquantum scutum, in ipsam albedinem" (Meister Eckhart, 1936b: 179). 
análogamente, el de la determinación creatural — no contiene la necesidad de la existencia: "ni tiene un algo de blancura en absoluto ni tampoco refluye nada - en cuanto escudo- hacia la blancura misma". La creatura, en tanto creatura, esto es, en tanto determinación, no posee existencia (esse). Esta le es dada por el ser, en la analogía propuesta, por la blancura. Así, una creatura que es, en tanto que es, recibe todo su ser a través del ser.

Parecería aquí estar presente la doctrina asumida por la escolástica de la escisión entre esencia y existencia ${ }^{41}$ : si asociamos de forma más directa a la creatura con la essentia, en el sentido que esta le permite identificarse como siendo-algo, entonces podemos sostener que ninguna determinación esencial contiene la necesidad de la existencia. Así, la creatura no podría darse a sí misma el ser, pues no se encuentra en su concepto. De esta manera, Dios-causa dona el esse. Y, precisamente, este "esse" (que est Deus) es condición de posibilidad para que las creaturas sean.

Este punto, el hecho de que Eckhart quiere señalar que las causas segundas solo inciden en la determinación creatural, pero no en la existencia de la creatura, se ve de forma clara cuando vuelve a pronunciar lo que dijo al comienzo del Prólogo: "todo compuesto, por ejemplo, una piedra, tiene el ser-piedra a partir de la forma de la piedra, pero el ser en sentido absoluto a partir de Dios solo en cuanto causa primera" ${ }^{\prime 2}$.

En relación a esto es que clarifica el movimiento que se da entre las causas. Pues señala que se dice que varias causas actúan sobre una misma sustancia y, con todo, nadie imagina distintos seres sobre los que aplican las distintas causas, sino que se comprende que refieren a aspectos distintos de un mismo ser: "un mismo ser, íntegramente, a partir del solo fin solo que finalmente, a partir de la forma, formalmente, y a partir de la materia, pasiva o receptivamente" 43 .

El propio Eckhart sostiene que este mismo mecanismo debe entenderse que ocurre en relación a la causa primera y las causas segundas, solo que "es necesario pensarlo en muchísima mayor medida acerca de

41 Eckhart expone en el Com. Al Éxodo 3,14 que su fuente para esta proposición es Avicena: "Quod quotiens fit, purum esse et nudum esse significat in subiecto et de subiecto et ipsum esse subiectum, id est essentiam subiecti, idem scilicet essentiam et esse, quod soli deo convenit, ciuis quiditas est sua anitas, ut ait Avicenna, nec habe quiditatem praeter solam anitatem, quam esse significat" (Meister Eckhart, 1992).

42 "Sic etiam potius totum compositum, puta lapis, habet esse lapidis a forma lapidis, esse vero absolute a solo deo, utpote a prima causa" (Meister Eckhart, 1936b: 180).

43 "Adhuc autem non est imaginandum quod causae rei -efficiens puta, finalis, formalis et materialis- singular esse afferent et conferant composito, sed res se tota cum omnibus suis partibus et proprietatibus idem esse a solo fine totaliter finaliter solum, a forma vero formaliter, a materia passive sive receptive" (Meister Eckhart, 1936b: 180). 
toda causa respecto de la primera y suprema causa de todas las cosas, que es Dios": ${ }^{44}$ así como diversas operaciones se dan sobre un mismo efecto; de la misma manera, la misma causa, Dios en su primer aspecto, puede operar de distintos modos, eficiente (causa primera) o formal (causa segunda).

Concluye, entonces, diciendo que:

Aunque las formas den el ser esto o aquello, ningún ente este o aquel da el ser - en cuanto esto o aquello, no en cuanto es -45 .

Clarificar que las causas segundas no pueden donar el ser vuelve a señalar un punto central del Prólogo: que solo Dios, in quantum causa eficiente, puede donar el ser (esse) a las creaturas. Esto es, puede hacer que pasen del no-ser al ser. Esto también puede leerse en las cuatro proposiciones antes mencionadas. En esta sección analizamos el sentido de estas proposiciones desde la perspectiva del esse, esto es, del efecto y, así, observamos que el esse - ens es la estructura del mundo finito (primera proposición), que el esse absoluto es el fundamento de las creaturas porque es la existencia misma (segunda proposición); que el esse es el efecto inmediato de la causa primera y que no hay ningún intermediario y, así, todo es (en) el esse (tercera proposición); y, finalmente, que la determinación se inscribe en entidades que ya existen, lo que muestra que la existencia es lógicamente anterior a la esencia en los entes creados (cuarta proposición).

Sin embargo, como cierre, también podemos atender a lo que estas proposiciones pueden expresar si las analizamos desde la perspectiva de la causa primera. Y, así, vemos que solo la causa primera puede donar el ser, puesto que ebulle, sale de sí y se constituye en efecto de sí misma (primera proposición), al ser causa primera, a partir de Dios todo es (segunda proposición), es causa primera inmediata puesto que no acepta intermediarios, lo que se refleja prístinamente en el movimiento de ebullición tal que Dios causa es Dios efecto (tercera proposición) y las causas segundas no adicionan ser, sino que, por el contrario, lo limitan al construir entidades compuestas (cuarta proposición). Así entonces, se hace presente la conocida sentencia eckhartiana: "Por fuera del ser y sin el ser todas las cosas son nada, también las cosas hechas" (Meister Eckhart, 1936b: 178).

\footnotetext{
44 "Hoc autem potissime necessarium est sentire de omni causa respectu primae et supremae causae omnium, quae est deus" (Meister Eckhart, 1936b: 181).

45 "Igitur nichil ens hoc vel hoc dat esse, quamuis forte dent esse hoc aut hoc, inquantum hoc aut hoc, non autem inquantum esse" (Meister Eckhart, 1936b: 178).
} 
Una reformulación de esto se encuentra también en su Comentario al Éxodo, cuando señala que: "todo ser creado concebido apartado, como distinto en sí mismo de Dios no es un ser, sino que es nada. Lo que es separado y distinto de Dios, es distinto y separado de la existencia"46. Pues Dios-causa crea al esse, que es Dios-efecto, base existencial de todo lo que es.

\section{CONCLUSIÓN}

Este artículo buscó estructurar la propuesta metafísica eckhartiana del mundo creado a través de la exposición de tres niveles lógicos del esse y observar su aplicación efectiva al interior del Prólogo a la obra de las proposiciones, al considerarla una obra que presenta una esquematización bastante clara del orden del mundo en el pensamiento eckhartiano y, por ello, se mencionó la hipótesis de Quero Sánchez señalando a este Prólogo como, en rigor, el Tratado acerca de ser, lo que es y la nada.

Como corolario, la exposición del esse como base fundante de todo ens hoc expresa no solo la metafísica eckhartiana, sino que abre a la consideración de las relaciones que se instituyen entre las propias creaturas y, por excelencia, entre los seres humanos. Plantear que toda creatura existe en virtud de Dios, más aún, que ese "la creatura es" encierra a Dios, que el solo término "creatura" se sostiene en Dios expone profundos lazos al interior del mundo creado debido a que expresa que la estructura íntegra del mundo es, en sentido fuerte, Dios. Y es aún más relevante que, entonces, cuando un esto se determina lo que ocurre es que se separa de esa totalidad unitaria que en el fondo cada creatura es, justamente, porque es. Si Dios es una unidad absoluta -que lo contiene todo-, cuando una creatura se determina traza una distancia con esa absolutez. Ahora bien, en tanto el fundamento de cada creatura es esa perfección, entonces, al determinarse, cada creatura no es todo lo otro que podría ser. Esto expresa, como se dijo, a la vez la multiplicidad entre las creaturas y con respecto a Dios mismo: Dios es todo en una absoluta simplicidad, y, así, no se encuentra privado de nada. Sí sucede esto con las creaturas, que se encuentran privadas de todo aquello que no son. Con esta premisa es que se mueve toda la obra alemana del dominico, llamando a abandonarse a uno mismo, a la determinación, para hacerse uno con la divinidad.

Sin embargo, esta lectura no solo puede operar, digamos, de forma "vertical/inmanente", buscando a Dios en uno mismo, en esa clave agus-

\footnotetext{
46 "Omne autem ens creatum acceptum vel conceptum seorsum per se distinctum a deo non es tens, sed est nihil. Separatum enim et distinctum a deo separum est et distinctum ab esse" (Meister Eckhart, 1992: 45).
} 
tiniana distinguible en Eckhart, sino que también puede volcarse como clave de análisis de la vida en comunidad: cada creatura y, en particular, los seres humanos somos todos algos determinados y, como tales, separados unos de otros; sin embargo, esa separación se fundamenta precisamente en que no somos todos esos otros existentes (y posiblemente existentes). Esto es, se conforma una relación que no solo nos coloca como efectos de una misma causa, sino que, puesto que esa causa nos constituye realy sustantivamente, nuestra especificidad descansa en no ser ningún otro ser humano que podría ser y que, en cierto sentido, reposa en cada uno.

De esta manera, el esquema metafísico eckhartiano no solo es una estructura especulativa interesante, sino que se abre a la consideración de propuestas de índole ética, que eleven el carácter de comunidad amparada en que necesitamos, ontológicamente, a los otros para ser.

\section{REFERENCIAS}

Bara Bancel, S. (2015). Teología mística alemana. Estudio comparativo del "Libro de la verdad" de Enrique Suso y la obra del Maestro Eckhart (Beiträge zur Geschichte der Philosophie und Theologie des mittelalters, NF 78). Münster: Aschendorff.

Beierwaltes, W. (1992). Primus est dives per se. Meister Eckhart und der Liber de Causis. En E.P. Bos \& P.A. Meijer (eds.), On Proclus and his Influence in Medieval Philosophy (pp. 141-169). Leiden: Brill.

Beierwaltes, W. (1972). Platonismus und Idealismus. Frankfurt am Main: Verlag V. Kostermann.

Caputo, J. (1974). Meister Eckhart and the Later Heidegger. Journal of the History of Philosophy, 12(4), 479-494.

D’Amico, C. \& Ludueña, E. (2018). Maestro Eckhart. Prólogo a la obra de las proposiciones. Revista española de filosofía medieval, 25, 219-229.

Lanzetta, B. (1992). Three Categories of Nothingness in Eckhart. The Journal of Religion, 72(2), 248-268.

Mayer, R. (2017). The Knowability of Divine Being according to Meister Eckhart's Principal Thesis: 'the act to be is God'. Alpha Omega, 20(3), 509583.

Meister Eckhart (1936a). Prologi in Opus tripartitum (LW I.1). Stuttgart: Kohlhammer.

Meister Eckhart (1936b). Prologus in Opus propositionum (LW I.1). Stuttgart: Kohlhammer.

Meister Eckhart (1992). Expositio Libri Exodi (LW II). Stuttgart: Kohlhammer.

Meister Eckhart (1994). Expositio sancti Evangelii secundum Iohannem (LW III). Stuttgart: Kohlhammer.

Meister Eckhart (2007). Quaestiones Parisienses (LW V). Stuttgart: Kohlhammer. Meister Eckhart (2015). Expositio Libri Genesis (LW I.1). Stuttgart: Kohlhammer. Mojsisch, B. (2001). Meister Eckhart. Analogy, Univocity and Unity (Transl. by O. F. Summerell). Amsterdam/Philadelphia: B.R. Grüner Publishing Company. 
Quero Sánchez, A. (2016). Meister Eckhart's treatise on Being, what is, and nothing, and the relationship between his commentaries on Genesis (Expositio on Genesis and Book of the Parables of Genesis). Revista Española de Filosofía Medieval, 23, 259-290.

Sanabria, C. A. (2015). Camino sin camino. Una reflexión filosófica actual desde la mística de Eckhart y Silesius. Veritas, (33), 159-181.

Sturlese, L. (2012). Eckhart as Preacher, Administrator, and Master of the Sentences. From Erfurt to Paris and Back: 1294-1313. The Origins of the Opus tripartitum. En J. M. Hackett (Ed.), A companion to Meister Eckhart (pp. 125-136). Leiden: Brill.

Tsopurashvili, T. (2012). The Theory of the Transcendentals in Meister Eckhart. En J. M. Hackett (ed.), A companion to Meister Eckhart (pp. 185-203). Leiden: Brill. 